\title{
ANALISIS SISMICO DE UNA ESTACION ELEVADA DEL METRO DE LA CIUDAD DE MEXICO
}

\author{
Por: Ing. Manuel Díaz Canales \\ Ing. Ricardo Pérez Ruíz. ${ }^{2}$
}

\section{RES U MEN}

La Línea 4 del Metro de la Cd, de México está resuelta casi en su totalidad por una estructura elevada constituída por trabes de concreto postensa das que, en tramos independientes, se apoyan libremente sobre columnas de con creto aisladas.

En este trabajo se presenta el análisis sismico de la estructura para un tramo de Estación, idealizada como un péndulo invertido. El análisis se hizo empleando los métodos estático y dinámico establecidos por las normas del Reglamento de Construcciones para el Ditrito Federal. En ambos métodos se tomó en cuenta el efecto que la inercia rotacional de la masa superior in duce a la estructura $y$, adicionalmente, en el análisis dinámico se estudió la influencia que ejerce la interacción suelo-estructura en la evaluación de los periodos naturales de vibración y, por 10 tanto, en la respuesta sismica. Los resultados obtenidos por cada uno de los métodos, se comparan con los -de pruebas de vibración forzada, realizacias por el Instituto de Inaeniería de la UNAM, y se concluye que, el análisis dinámico de un voladizo con inercia rotacional de la masa superior e interacción suelo-estructura es el más conveniente para fines de diseño.

(1) Vicepresidente del grupo ICA.

(2) Subjefe del Departamento de Estructuras, ISTME, S.A. 


\section{INTRODUCCION}

Durante la segunda etapa de las ampliaciones del Metro de la Ciudad de México (1978-1982), se realizó la construcción de la Línea 4 con una longitud total de $10.75 \mathrm{~km}$ que recorre las Avenidas Inguarán y Fco. Morazán desde la colonia Martín Carrera, en el norte, hasta el barrio de Santa Anita, en el sur.

Una de las características más notables de esta Linea es que casi la totali-dad de su estructura es elevada, alojando a las pistas a una altura típica de $8.10 \mathrm{~m}$ sobre el nivel de la banqueta.

Incluye a 10 largo de su recorrido 10 estaciones, de las cuales 8 son eleva-das y 2 superficiales; del mismo modo, comprende $9.12 \mathrm{~km}$ de tramo elevado y $1.63 \mathrm{~km}$ de tramo superficial.

Con el objeto de conservar el mayor número de carriles para vialidad, se optó por utilizar una sola hilera de columnas de apoyo, ya que esto permite redu-cir el ancho del camellón. Una vez definido el apoyo en una sola hilera de columnas, se estudiaron varias alternativas posibles para las trabes, tanto desde el punto de vista del material a emplear como desde el punto de vista de la sección transversal más conveniente y de los claros que podrian salvarse. Se anąlizó también la posibilidad de usar estructuras continuas o simple mente apoyadas.

Las estructuras continuas presentaban ventajas de distribución de elementos mecánicos; sin embargo, para su correcto funcionamiento se debía procurar que los apoyos no permitieran desplazamientos verticales diferenciales que ocasio narian esfuerzos adicionales de importancia. Para lograr esto, se requeria que, debido a las condiciones del subsuelo sobre el que se desplanta la es--tructura, la cimentación se apoyara sobre pilotes de punta, lo que motivaría que se afectara la zona adyacente a la cimentación. Además, las ventaias de la continuidad solo serian en la dirección de la Linea, pues en la dirección perpendicular, la estructura sería isostática. Debido a estas razones se optó por utilizar la solución con tramos libremente apoyados, que tolera hundimientos diferenciales.

La Línea en estudio se clasificó en su totalidad dentro de la zona III (Ref.1) altamente compresible y, considerando la importancia que reviste el hundimiento regional en el valle de la Ciudad de México, fué necesario adoptar una cimentación tal que "siguiera" dicho hundimiento y se estudiaron varias alternativas, desde el uso de cajones compensados, hasta el de zapatas semicompensadas con pilotes de fricción, que fué la solución finalmente adoptada.

\section{DESCRIPCION DE LA ESTRUCTURA.}

En 10 que se refiere a las trabes, se decidió utilizar trabes de sección ca-jón de concreto postensado colado en sitio, libremente apoyadas sobre las columnas, con claros entre ellas de $35 \mathrm{~m}$ en los tramos de la Línea y de $25 \mathrm{~m}$. en las estaciones.

En las estaciones (fig. 1), la sección transversal de la trabe es de $2.20 \mathrm{~m}$ de peralte. Está formada por 4 nervaduras de $40 \mathrm{~cm}$ de espesor cada una, una losa superior de $14.50 \mathrm{~m}$ de ancho con el objeto de alojar a dos andenes laterales $y$, una losa inferior de $8.90 \mathrm{~m}$ de ancho. La losa superior se une con la inferior por medio de paredes inclinadas. Para mejorar la rigidez torsio- 
nal de la trabe y uniformizar los desplazamientos verticales se colocaron dos diafragmas extremos y 4 intermedios.

Las trabes se apoyaron sobre placas de neopreno, una de ellas rigida horizontalmente para restringir los desplazamientos y la otra flexible, equivalente a un apoyo móvil. Para restringir los movimientos de la trabe en dirección perpendicular a la Linea, se usó una saliente que encaja en un hueco dejado previamente en la columna.

Las dimensiones de la columna en el extremo superior están regidas por los es pacios entre las trabes para poder postensarlas y por la separación entre apo yos de neopreno (orientados en dirección perpendicular a la Línea) para tomar el momento de volteo producido por el sismo. En la base de la columna, la -sección que resultó por requisitos de resistencia fué de menores dimensiones que en el extremo superior, de tal modo que, la columna quedó de sección va-riable.

La cimentación está formada por una zapata cuadrada de $14 \mathrm{~m} \times 14 \mathrm{~m}$ con espe-sor de $1.40 \mathrm{~m}$ y un dado de repartición de carga de $4 \mathrm{~m} \times 4 \mathrm{~m}$ y de $80 \mathrm{~cm}$ de es pesor. El número de pilotes de fricción varía de 26 a 32 dependiendo de la capacidad de carga de éstos. En las esquinas de las zapatas se dejaron prepa raciones para colocar pilotes de control a futuro cuya función será la de enderezar la estructura en caso de que fuera necesario.

La estación asi estructurada, resulta altamente sensible a los efectos sismi$\cos , y$ es motivo de este trabajo presentar los métodos de análisis sísmico em pleados, así como los comentarios por los que se definió el más conveniente para fines de diseño:

MASA, INERCIA ROTACIONALY PROPIEDADES ELASTICAS DE LA COLUMNA.

En el análisis de un péndulo invertido, la masa del extremo superior de la co lumna y su inercia rotacional tienden a producir un desplazamiento y un airo, respectivamente, en ese extremo. Los valores de la masa e inercia rotacional considerados en nuestro caso, fueron estimados tomando en cuenta las carcas muertas y vivas en ambas direcciones de análisis.

La carga muerta está dada por los siguientes elementos: peso propio de la tra be; cargas adicionales sobre la trabe tales como el balasto, durmientes, via, instalaciones, andenes, cubierta y peso tributario de la columna al extremo superior.

Las cargas vivas que se tomaron en cuenta para el análisis, son las produci-das por los trenes de carga tipo y de mantenimiento. Para determinar las com binaciones de los trenes que producen efectos más desfavorables se estudiaron diferentes posiciones de ellos y se seleccionaron las que producen mayor descarga vertical y mayor flexión en ambas direcciones de análisis, dando por re sultado 4 combinaciones diferentes.

Para la combinación de cargas permanentes (carga muerta y viva), se consideró el peso de los trenes tipo y de mantenimiento incrementado por impacto en $30 \%$ y $25 \%$ respectivamente. Para valuar la fuerza horizontal transversal, se tomó la producida por el cabeceo de uno de los 2 trenes (el más desfavorable) y la fuerza longitudinal se calculó como la suma de la acleración de uno de 1os .trenes más la desaceleración (frenaje) del otro tren circulando en sentido -- 
contrario.

Para la combinación de cargas accidentales (carga muerta, viva y sismo) sరlamente se consideró el peso de los trenes sin incrementar por impacto, en la dirección transversal el cabeceo de uno de los trenes y en la dirección longi tudinal el frenaje o la acleración también de uno de los 2 trenes (el más des favorable).

En la tabla $\mathrm{T}-1$ se resumen los valores de masa e inercia rotacional para las cuatro combinaciones de carga muerta y viva en ambas direcciones de análisis.

Las propiedades elásticas de la columna, tales como la rigidez por traslación y por rotación, se obtuvieron integrando las ecuaciones que dan los desnlazamientos y giros en el extremo libre de la columna, bajo la acción de una carga horizontal y un momento flexionante, unitarios, aplicados en ese punto. En la figura 2 se explican detalladamente estas rigideces y en la tabla T-2 se resumen los valores obtenidos.

\section{METODOS DE ANALISIS SISMICO.}

Para la estimación de las fuerzas sísmicas bajo las que se verá sujeta la estructura, se emplearon los métodos Estático y Dinámico modal que presenta el Reglamento de Construcciones para el Distrito Federal (Ref. 1). En ambos métodos se tomó en cuenta el efecto de la inercia rotacional de la masa en el extremo superior de la columna. Además, en el análisis dinámico, se consideró la interacción suelo-estructura para la evaluación de los períodos naturales de vibración.

Conforme al RCDF, las consideraciones que se hicieron para diseño por sismo son las siguientes:

Según su destino, la estructura para el Metro elevado se clasifica dentro del grupo $A$, ya que es una construcción cuyo funcionamiento es muy importante bajo la acción de un sismo y oue en caso de fallar causaría pérdidas directas o indirectas excepcionalmente altas en comparación con el costo necesario para aumentar su seguridad.

Segün su estructuración corresponde al tịno 1, siendo un péndulo invertido ya que más del $50 \%$ de su masa se encuentra en el extremo superior y cuenta con. un sólo elemento resistente en la dirección de análisis.

De acuerdo con el artículo 234, se tomó el valor del coeficiente sísmico "c" indicado para la zona III (terreno compresible) $c=0.24 \times 1.3=0.312$. Para obtener las fuerzas sísmicas de diseño, tanto por el método estático como por el dinámico, el coeficiente sísmico y las ordenadas de los espectros de diseño se redujeron en función de la ductilidad que puede desarrollar la estructu ra. Para definir el factor de ductilidad, Q, la estructura corresponde al caso 3 del articulo 235 del citado Reglamento, ya que la resistencia contra fuerzas laterales es suministrada por columnas de concreto, y no se cumple 10 especificado en el caso 2 , en el que es necesaria la presencia de marcos que, sin la ayuda de muros o contravientos, sean capaces de resistir cuando menos el $25 \%$ de la fuerza cortante total. Por tanto, nara el caso que nos ocupa se tomö $Q=2$.

Las deformaciones se calcularon multiplicando por Q las causadas por las fuer zas sísmicas reducidas. 
La estructura se analizó bajo la acción de dos componentes horizontales ortogonales del movimiento del terreno. Los efectos correspondientes (desplaza-mientos y fuerzas internas) se combinaron con los producidos por las fuerzas permanentes. Dicha combinación se hizo sumando vectorialmente los efectos de las fuerzas permanentes, los que resultaron al actuar el sismo en una direc-ción y el $50 \%$ de los de la otra dirección de análisis.

El factor de carga F.C. para diseño por resistencia última se consideró, se-gún el artículo 237 inciso VIII, 20\% superior al especificado en el artículo 220 ya que se cuenta con un sólo elemento para resistir los efectos sismicos; asi, F.C. $=1.1 \times 1.2=1.32$.

Para la aplicación de los análisis estático y dinámico se utilizó el espectro de diseño sísmico que presenta el RCDF en su articulo 236 para estructuras -del Grupo A desplantadas en terreno compresible. En la figura 3 se muestran los valores que lo definen.

\section{ANALISIS ESTATICO.}

De acuerdo con el artículo 240 del RCDF, para calcular la fuerza cortante se supuso una fuerza horizontal actuando sobre el punto donde se considera con-centrada la masa (extremo superior de la columna o centro de rotación de la masa (R). Dicha fuerza se tomó de tal manera que la relación $V_{\hat{\delta}} / W$ en la base fuera igual a $c / Q$ pero no menor que $a_{0}$, es decir:

$V_{\hat{\sigma}} / W=c / Q>a_{0}$, de donde

$V_{\hat{0}}=c W / Q$

En las expresiones anteriores $V_{\theta}$ es la fuerza cortante en la base, $W$ el peso de la masa superior, c el coeficiente sismico ya definido, $Q$ el factor de reducción por ductilidad y $\mathrm{a}_{\circ}$ es la acleración del terreno que, por tratarse de una estructura del grupo A desplantada en terreno compresible, se tomó igual a $0.06 \times 1.3=0.078$ (ver figura 3 ).

En el análisis de la estructura, además de la fuerza lateral se tomaron en -cuenta las aceleraciones verticales de la masa superior asociadas al giro de dicha masa con respecto a un eje horizontal perpendicular a la dirección de análisis. Este efecto fué más importante en la dirección transversal a la Lí nea y se tomó equivalente a un par aplicado en el extremo superior de la co-lumna cuyo valor es, conforme al artículo 240-III:

$M_{\hat{o}}=\frac{1.5 V_{\hat{o}} r_{0}^{2} 0_{0}}{\delta_{0}}$

donde $r_{0}$ es el radio de giro de la masa $y$ es igual a $\sqrt{J / m}, \theta_{0}=v_{0} \gamma$ es el gi ro del extremo superior de la columna bajo la acción de la fuerza lateral $V_{\hat{o}}$, $\delta_{0}=V_{\hat{o}} / K$ es el desplazamiento lateral de dicho extremo bajo la acción de $V_{\hat{o}}^{\circ}$.

Conforme el artículo 240-II, los elementos calculados $V_{\hat{o}}$ y $M_{\hat{c}}$ se redujeron por efecto de la influencia del periodo fundamental de vibración de la estruc tura. En la estimación del periodo fundamental se tomaron también en cuenta los efectos de la inercia rotacional, modificando la fórmula que propone el RCDF en la siguiente forma: 
40

$T=6.3 \sqrt{\frac{m \delta_{1}^{2}+1 \theta_{1}^{2}}{V_{\hat{o}} \delta_{1}+\frac{1}{M_{\hat{o}} \theta_{1}}}}$

siendo $\delta$, y $\theta_{1}$, los desplazamientos totales al actuar $V_{\hat{o}}$ y $M_{\hat{o}}$ simultáneamente, es decir:

$\delta_{1}=V_{\hat{o}} / K+M_{\hat{o}} \gamma$

$\theta_{1}=V_{\hat{o}} \gamma+M_{\hat{o}} / K_{r}$

Los términos $K, K_{r}$ y $\gamma$ se definen en la figura 2.

Por tratarse de una estructura rígida desplantada en suelo compresible, el período fundamental calculado, $T$, resultó menor que el primer período dominante del terreno, $T_{1}$, por lo que, la fuerza cortante reducida $V$, se valuó con la si guiente fórmula:

$V=\frac{\text { amg }}{Q^{\prime}}$, donde

$a=a_{0}+\left(c-a_{0}\right) T / T_{1}$

$Q^{\prime}=1+(Q-1) T / T$,

$y$ el momento reducido, $M$, con:

$M=\frac{1.5 V r_{0}^{2} \theta}{\delta}$

donde $\theta=V \gamma$ y $\delta=V / K$.

Las deformaciones finales se calcularon multiplicando por 0 las causadas por las fuerzas sísmicas reducidas, es decir:

$\Delta=(V / K+M \gamma) Q$

En la tabla T-3 se resumen los valores obtenidos de $V, M$ y $\triangle$ rara las 4 combinaciones de carga viva consideradas.

ANALISIS DINAMICO.

Para el análisis dinámico se consideraron tres casos: voladizo con masa concen trada en el extremo y suelo infinitamente rígido, voladizo con masa con iner-cia rotacional en el extremo y suelo infinitamente rigido y voladizo con masa con inercia rotacional en el extremo e interacción suelo-estructura. Para es te análisis se usaron los modelos propuestos en la ref. 2 suponiendo comnortamiento lineal.

El modelo más simple es en el que se considera la masa concentrada en el extre mo superior de la columna y la base empotrada. Para este caso habrá un sólo grado de libertad en traslación horizontal y el periodo de vibración está dado por la expresión

$T=2 \pi \sqrt{\mathrm{m} / \mathrm{K}}$

donde $m$ es la masa concentrada en el extremo superior y $K$ es la rigidez al desplazamiento del extremo superior de la columna.Con el periodo calculado se obtuvo la ordenada de aceleraciones del espectro de diseño, a, y el factor de ductilidad reducido $Q^{\prime}$ correspondiente. El valor de la fuerza cortante se de- 
determinó con la expresión $V=a m g / Q^{\prime}$, en la que g es la aceleración de $1 a-$. gravedad. En este caso, el momento en el extremo superior fué nulo. Las deformaciones, $\Delta$, se calcularon con la misma expresión que para el análisis es tático. En la tabla $T-3$ se resumen los valores obtenidos de $V$ y $\Delta$ para las cuatro combinaciones de carga viva.

En el modelo que considera la inercia rotacional de la masa superior y la base empotrada, se presentan dos grados de libertad: el desplazamiento horizon tal y el giro en el extremo superior de la columna; por tanto, habrá dos mo-dos de vibración. En la ref. 3 se demuestra que la frecuencia circular natu ral de vibración, $\omega_{n}$, para el modo de vibración $n$, se puede valuar con la expresión:

$\omega_{n}^{2}=\frac{\rho^{2}+\Omega^{2}}{2 k} \pm \frac{1}{2} \sqrt{\left(\frac{\rho^{2}+\Omega^{2}}{k}\right)^{2}-\frac{4 \rho^{2} \Omega^{2}}{k}}$

en la que $\rho^{2}=\mathrm{K} / \mathrm{m}$ es el cuadrado de la frecuencia circular natural por tras lacioón, $\Omega^{2}=\mathrm{Kr} / \mathrm{J}$ es el cuadrado de la frecuencia circular natural por rota-ción y $k=1-\theta \delta$ es un valor constante. Una vez conocida la frecuencia circular natural de vibración, se obtuvieron los perídos naturales, $t_{n}$, correspondientes a cada modo de vibración $n$, con la siguiente expresión:

$t_{n}=2 \pi / i i_{n}$

Las configuraciones modales se determinaron con (ref.3):

$\frac{x_{n}}{\varepsilon_{n}}=\frac{K \delta}{K\left(\frac{K}{K}-m \omega_{n}^{2}\right)}$

donde $X_{n}$ y $\varepsilon_{n}$ son los desplazamientos y giros totales, respectivamente, para cada modo de vibración.

A partir del periodo y de las configuraciones modales, se pueden determinar las ordenadas del espectro de acleraciones, los coeficientes de participación y las respuestas máximas de cada modo de vibración mediante las expresiones:

$v_{n}=\left|c_{n}\right| s_{a n} m x_{n}$

$M_{n}=\left|C_{n}\right| S_{a n} J \varepsilon_{n}$

donde $C_{n}$ es el coeficiente de participación para cada modo de vibración $n$, -$S_{a n}$ es la ordenada del espectro de aceleraciones reducida por ductilidad, $V_{n}$ y $M_{n}$ son, respectivamente, la fuerza cortante y el momento flexionante para ${ }^{n}$ el modo n. Finalmente, para el cálculo de la respuesta total, $R$, considerando la respuesta de cada modo, se usó la expresión:

$R=\left(\Sigma R_{j}^{2}\right)^{\frac{1}{2}}$

por 10 tanto:

$V=\sqrt{V_{1}^{2}+V_{2}^{2}}$

$M=\sqrt{M_{1}^{2}+M_{2}^{2}}$

en donde $V$ y $M$ representan la fuerza cortante $y$ el momento flexionante totales, 


\section{2}

aplicados en el extremo superior de la columna. Para el cálculo de las defor maciones se utilizó la epxresión $\Delta=(V / K+M Y) Q$. En la tabla $T-3$ se resu-men los valores obtenidos de $V, M$ y $\Delta$.

Si la estructura estuviera desplantada sobre terreno firme, el modelo descrito seria adecuado; sin embargo, se ha demostrado que en péndulos invertidos desplantados en terreno compresible, el fenómeno de interacción suelo-estructura puede ser importante en la respuesta (ref.4).

En este caso particular como la estructura resulta bastante rígida, sus pe--ríodos de vibración son menores que el primer periodo dominante del terreno y se cae en la rama ascendente del espectro de diseño, por lo que, como la in-teracción suelo-estructura alarga los periodos de vibración, las ordenadas es pectrales que se obtienen son mayores que cuando este efecto no se considera. En el modelo que considera la interacción suelo-estructura, las restricciones del suelo fueron idealizadas mediante resortes de comportamiento lineal, uno para desplazamientos lineales horizontales y otro para deformaciones angula-res de cabeceo de la cimentación (fig. 4).

Para obtener los valores de $K_{\text {y }}$ y $R_{\text {, }}$, que son las rigideces correspondientes a la traslación y rotación de ${ }^{C}$ la báse de la cimentación, se utilizó la teoría de Hrennikoff, considerando al grupo de pilotes como barras de longitud infini ta en un medio elástico continuo (ref. 5). El coeficiente de reacción de sub grado horizontal se tomó igual a $0.562 \mathrm{~kg} / \mathrm{cm}^{3}$ para pilotes de $50 \times 50 \mathrm{~cm}$ y la rigidez del pilote a cargas verticales instantáneas igual a $7500 \mathrm{ton} / \mathrm{m}$. Para el caso de 26 pilotes, la rigidez al desplazamiento lateral es del orden de $K_{c}=21300$ ton $/ m$, y la rigidez de giro es de $R_{c}=3330000$ ton-m/rad, para am̌bas direcciones de análisis.

Las rigideces correspondientes a la traslación y rotación de la cimentación,influyen en la evaluación de la frecuencia circular natural de vibración, "("n, $y$ en las configuraciones modales $x_{n} / \varepsilon_{n}$. Las expresiones que se emplearon para su cálculo son:

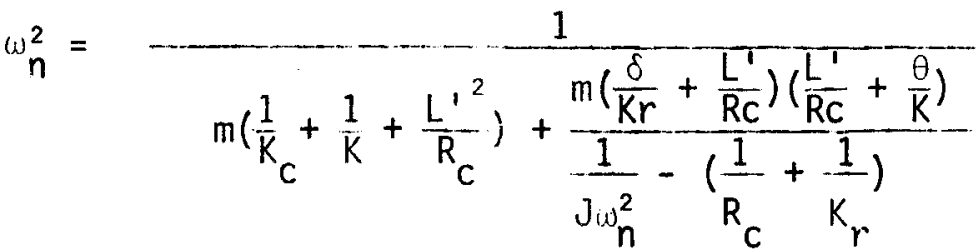

$$
\begin{aligned}
& \frac{X_{n}}{\varepsilon_{n}}=\frac{J\left(\omega_{n}^{2}\left(\frac{\delta}{K r}+\frac{L^{\prime}}{R c}\right)\right.}{1-m \omega_{n}^{2}\left(\frac{1}{K_{c}}+\frac{1}{K}+\frac{L^{\prime 2}}{R_{c}}\right)}
\end{aligned}
$$

en donde cada una de las variables se define en la figura 4 . Conocidos, 1os valores de la frecuencia, periodo y configuración modal para cada modo de vibración, la respuesta sísmica se obtuvo con las mismas expresiones que para el caso del voladizo con inercia rotacional y suelo infinitamente rígido. Las deformaciones se calcularon con la ecuación de desplazamiento lineal total afectada por el factor de ductilidad, o sea: 
$\Delta=\left[\frac{V}{K_{c}}+\frac{V}{K}+\frac{M \delta}{K_{r}}+\frac{\left(M+V L^{\prime}\right) L^{\prime}}{R_{c}}\right] Q$

en el extremo superior, y en la base de la zapata

$x_{0}=V / K_{c}$.

En la tabla T-3 se resumen los valores obtenidos por este criterio.

\section{COMPARACION DE RESULTADOS.}

Como se observa en la tabla T-3 y en la figura 5, la fuerza cortante horizontal, V, para la condición de carga II, se mantiene constante en cualquier nivel, siendo la más desfavorable la que corresponde al análisis considerando la interacción suelo-estructura y aproximadamente $7 \%$ y $26 \%$ mayor que 1 a obtenida por el método estático en las direcciones transversal y longitudinal, -respectivamente. En la corona de la columna el momento mayor corresponde al análisis estático; sin embargo, se observa que en la base de la zapata en la dirección longitudinal (fig. 6b) el momento mayor pertenece al análisis con interacción debido al incremento producido por la fuerza horizontal que actúa en el extremo superior. En la dirección transversal el momento que rige en todos los niveles es el estático, pero, el método que rige después del estáti co es el de interacción suelo-estructura. En la figura $6 a$ y $6 \mathrm{~b}$ se observa que el momento obtenido con el modelo de masa concentrada y suelo rigido nos da resultados similares a los que se obtienen al considerar la inercia rota-cional de la trabe; sin embargo, este modelo se analizó únicamente para conocer la magnitud de la fuerza cortante y sus efectos, ya que el RCDF no permite analizar péndulos invertidos sin considerar el efecto de la inercia rotacio-nal.

Si consideramos la superposición de efectos por flexión como lo especifica el RCDF, que consiste en tomar para diseño los efectos de sismo en una dirección más el $50 \%$ de los de la otra dirección de análisis, observaremos que, para la condición de carga II, los resultados del sismo en la dirección transversal más el $50 \%$ de la dirección lonqitudinal (fig. 7.a) obtenidos tomando en cuenta la interacción resulta $22 \%$ menores que los estáticos en la base de la zapa ta y $6 \%$ menores en 1 a dirección longitudinal (fig. 7.b), por 10 que se puede afirmar que el método estático da resultados conservadores, que justifican su empleo como un método simplificado de análisis.

Los desplazamientos finales que se obtuvieron al analizar el modelo con inter acción suelo-estructura resultan mucho mayores que los obtenidos con los -..otros dos modelos dinámicos y que el estático. Esto se debe, principalmente, a la consideración de airos y desplazamientos en la base de la estructura --(fig. 8).

Con el objeto de tener un conocimiento adecuado de la respuesta dinámica de la estructura elevada del Metro, el Instituto de Ingeniería de la UNAM realizó pruebas de vibración forzada sobre la columna CC-34 de la Estación "Canal del Norte", y como resultado de esta prueba se obtuvo un período asociado al modo fundamental de vibración de 0.17 seq. (ref.6). Los periodos estimados en los aná lisis estático y dinámico para la misma columna fueron los siguientes: $0.052^{-}$ seg. para el modelo de masa concentrada y suelo riqido, 0.045 seq. discretizan do a la columna en una serie de masas concentradas y suelo rigido, $0.153 \mathrm{seg}$. considerando la interacción suelo-estructura, siendo este valor el que más se 
aproxima al obtenido por las mediciones del Instituto de Ingenieria.

\section{COMENTARIOS FINALES Y CONCLUSIONES.}

Resumiendo los puntos más sobresalientes se tiene:

La estructura del Metro elevado es vulnerable a los sismos por estar apoyada en una sola columna que contiene más del $50 \%$ de la masa en el extremo supe--rior y porque basta la formación de una sóla articulación plástica para produ cir el colapso. Además, es una estructura rigida desplantada sobre suelo com presible, por 10 que puede presentarse interacción suelo-estructura importante. Este efecto alarga los periodos naturales de vibración, por lo que se ob tiene mayor ordenada en el espectro de diseño $y$, en consecuencia, mayor res-puesta sismica.

Al actuar el sismo en la dirección transversal, los momentos flexionantes son más criticos por el método estático; sin embargo, por ser un método simplifica do es conservador. Los efectos más desfavorables por el método dinámico se obtuvieron al considerar la interacción suelo-estructura (fuerza horizontal, flexión y desplazamientos).

De los tres valores calculados del periodo de vibración, el más cercano al re gistrado por el Instituto de Ingeniería corresponde al de interacción suelo-estructura.

Dada la importancia de la estructura y tomando en cuenta los puntos anterio-res se conciuyó que el efacto de la interacción suelo-estructura debía in--.cluirse en el diseño del Metro elevado.

REFERENCIAS.

1.- Manual de diseño por sismo según el Reglamento de Construcciones para el Distrito Federal. Publicación.406 del Instituto de Ingeniería, UNAM, ju1 io 1977.

2.- Rascón Châvez 0., Efectos sísmicos en estructuras en forma de péndulo in-vertido. Revista de la Sociedad Mexicana de Ingeniería Sísmica A.C. ---1965.

3.- Cárcamo Velázquez F.R. y Pérez Ruíz R. "Análisis Sísmico de la Línea Flevada del Metropolitano de la Ciudad de México", Tesis Profesional, Fac. de Ingeniería, UNAM, enero 1981.

4.- Newimark y Rosenblueth. "Fundamentos de Ingeniería Sismica". Ed. Diana.

5.- Teng W.C. Foundation Design. Pretice-Hall, Inc. 1962.

6.- Mena E. y Sandova1. Frecuencia fundamental del conjunto columna-dado-zapa ta-pilotes del tramo elevado del Metro. Proyecto 9145, Instituto de Ingeniería, UNAM, marzo 1980. 


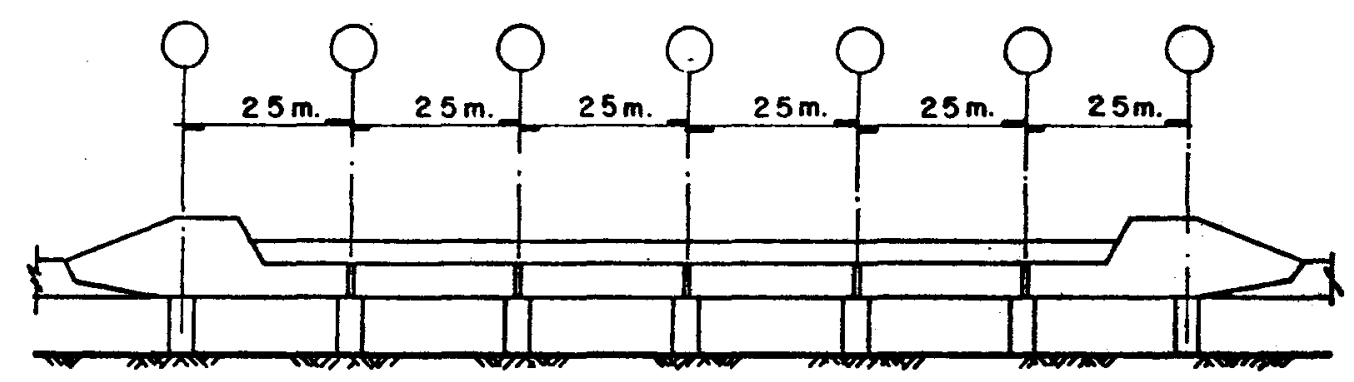

a) ELEVACION LONGITUDINAL
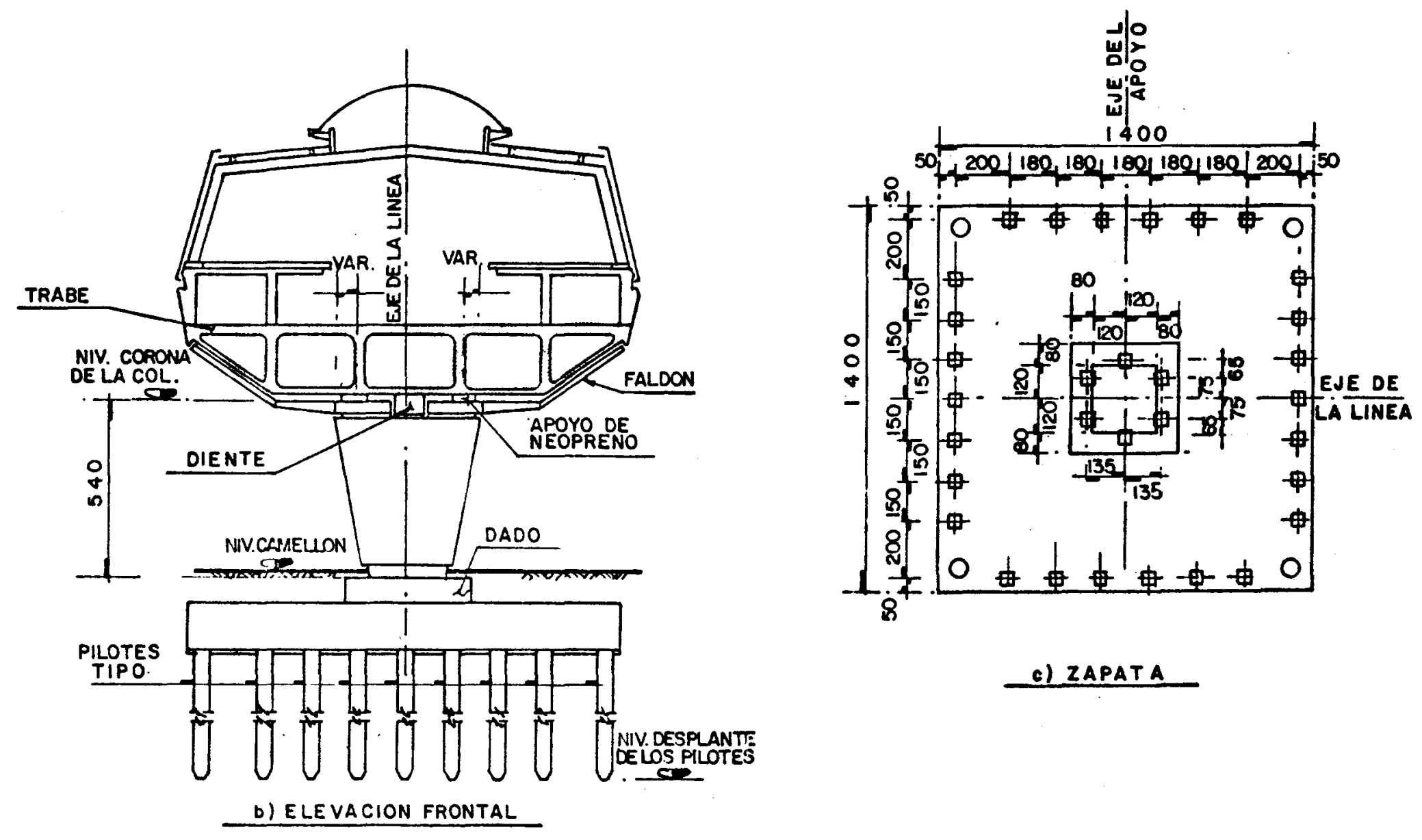

c) ZAPATA

Fig. 1.- DIMENSIONES GENERALES DE UNA ESTACION ELEVADA. 

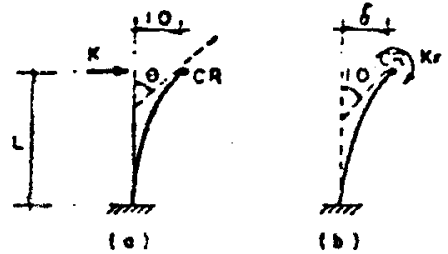

(b)

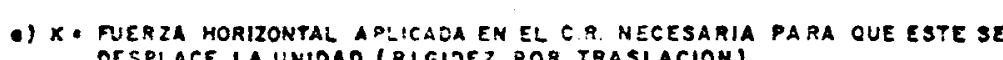

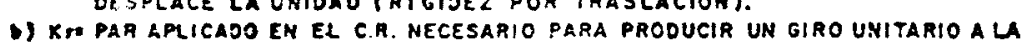
A:TURA DEL C.R. (RIGIOEZ POR POTACION).

C) O) $Y$. BOTACLON OE C. COUMVA AL ACTUAR UNA CARGA HORIZONTAL UNITARIA EN EL

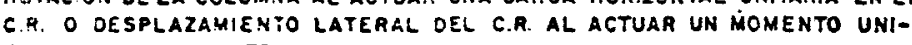

Q : GIRO EEL CR PRGJUCIDO POR LA FUERZA $K$.

8. DESPLAZAMIEnTO Lateral, OEL C.R. PRODUCIDO POR EL PAR $K$ I.

716. 2- DEFIMICION DE RIGIOECES DE UNA COLUMNA EN VOLADIzO

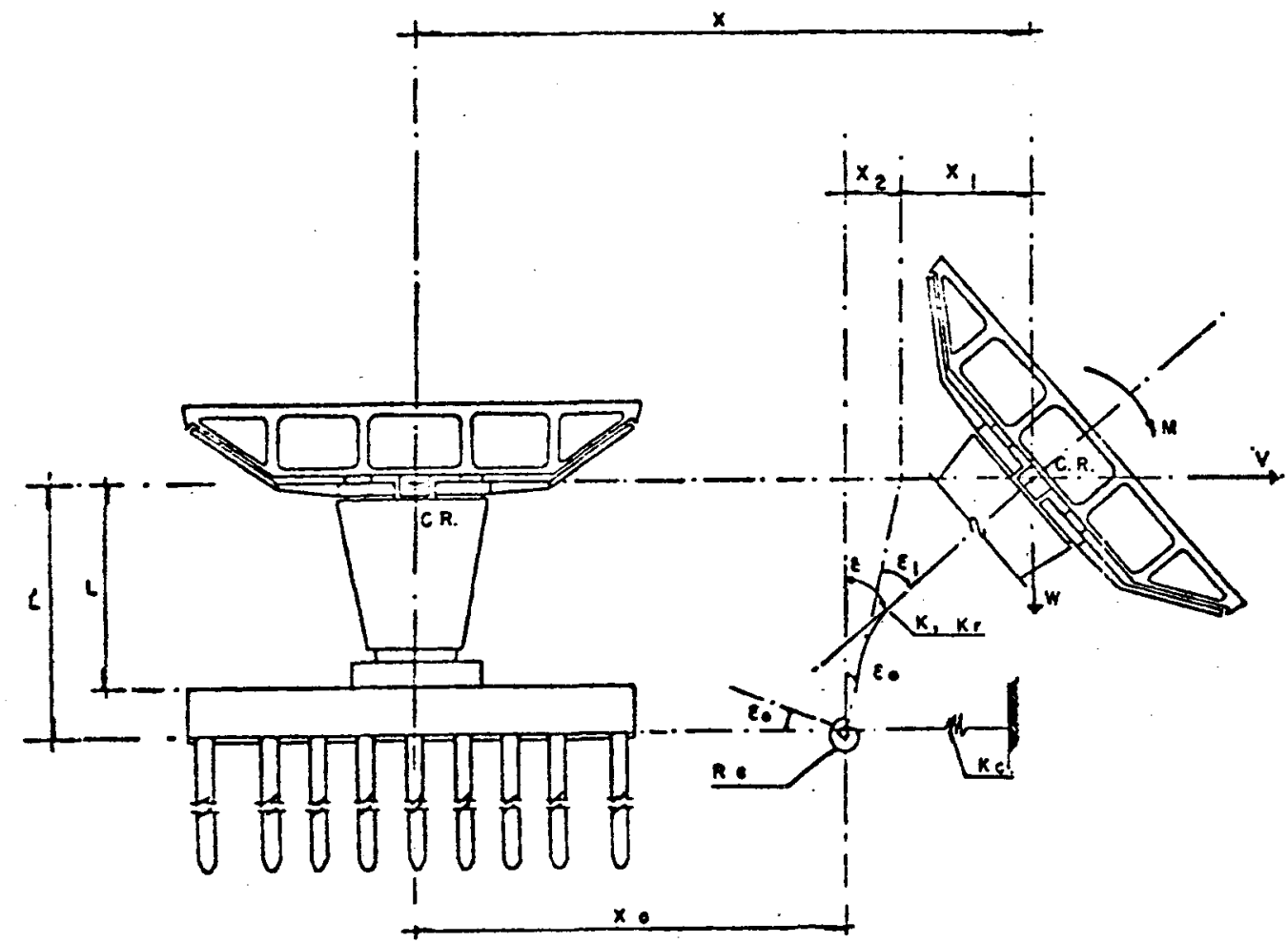

m - MASa sUPSRIOR.

- inercia rotacional oe la masa.

x fuerza horizontal aplicada en c r neeesaria para oue este se oesplace ia unidad.

kr - par aplicado en el c.r. necesario para producjo uh giro unitario a la altuga oel c. a.

Ke rigidez de la cimentacion al dezplazamiemto horizontal.

re - rigicez de la cimentacion al giro.

C.R. : CENTRO OE ROTACIOH.

- desplazamiento lateral del C.R. prooucioo pon el momento $\mathrm{K}$.

- - giro del c.r. producido por la fuerza $x$

$x_{0}$ - traslacion de la base.

$x_{2}$ - desplazamisnto lineal del c.a. producioo pon la rotacion oe la base.

$x$ - Desplazamiento lineal total del G.R

c. Rotacion de la base.

c oesplazhaiento angular total oel c.r. 


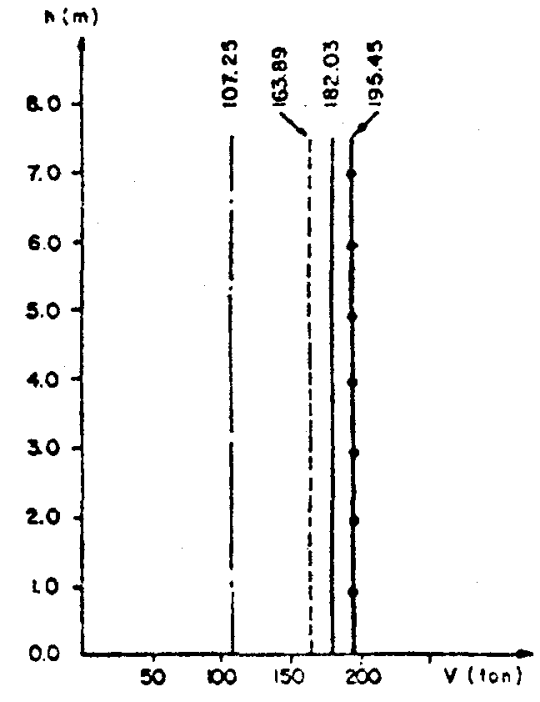

a) ANALISIS TRANSVERSAL

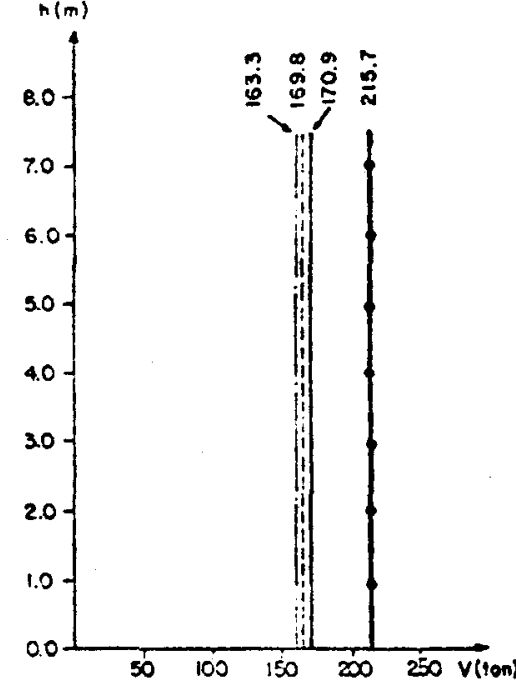

b) ANALISIS LONGITUDINAL

Fig. s diagrama de fuerzas cortantes para la conolcion de carga II
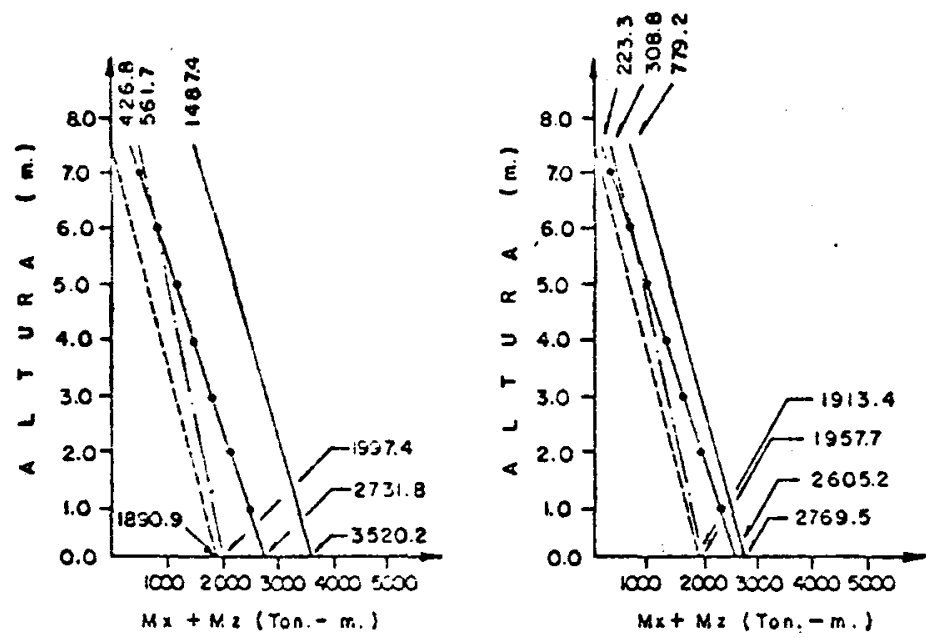

0)- SISMO TRLNSV.+ $50 \%$ SISM.O LONG.

b) $=30 \%$ SISMO TRANSV.+ SISMO LONO

FI6. 7-DIAGRAMA DE SUPERPOSICION DE MOMENTOS PARA LA CONDICION DE CARGA II.

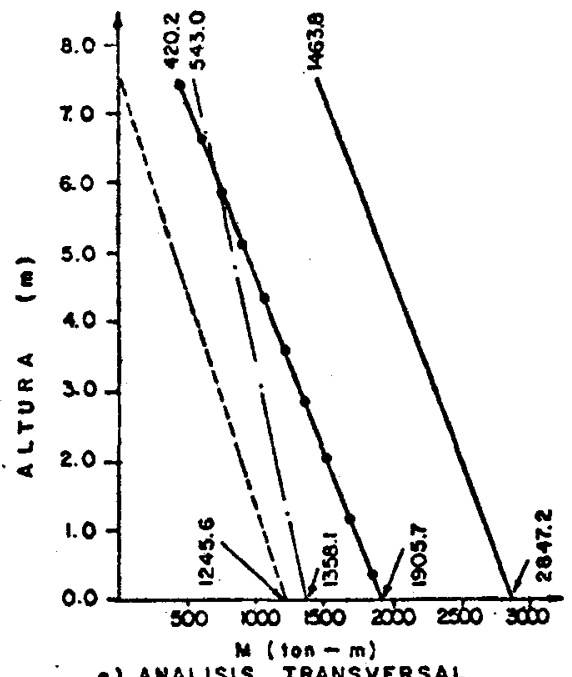

0) ANALISIS TRANSVERSAL

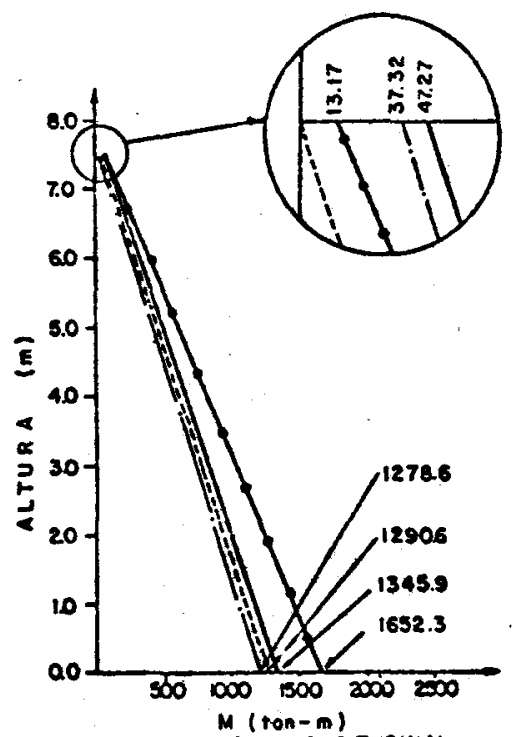

Fig. 6 diagrama de momentos para la condicion de carga $\boldsymbol{I}$.

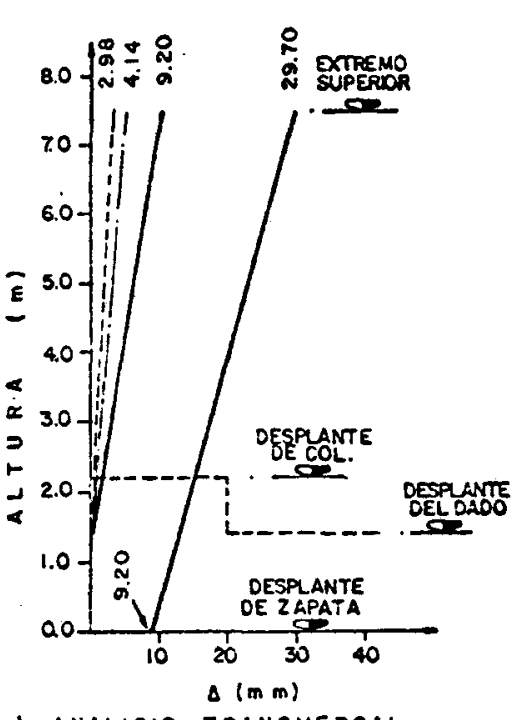

a) ANALISIS TRANSVERSAL

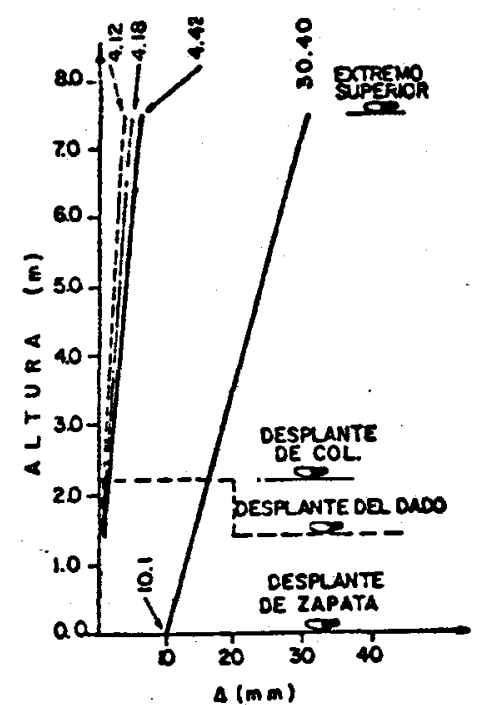

D) analisis longitudinal
ESTATICO

MASA CONCENTRAOA Y SLELO INFINITAMENTE RIGIOO

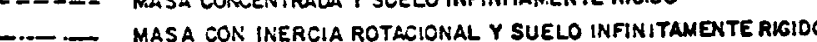

- MASA CON INEREIA ROTACIONAL Y SUELO INFNITANEITE RIIDO

Fig.6:- Desplazamientos finales para la condicion oE Carga II 
TABLA T-3. RESUMEN dE RESULTAdOS OBTENIDOS.

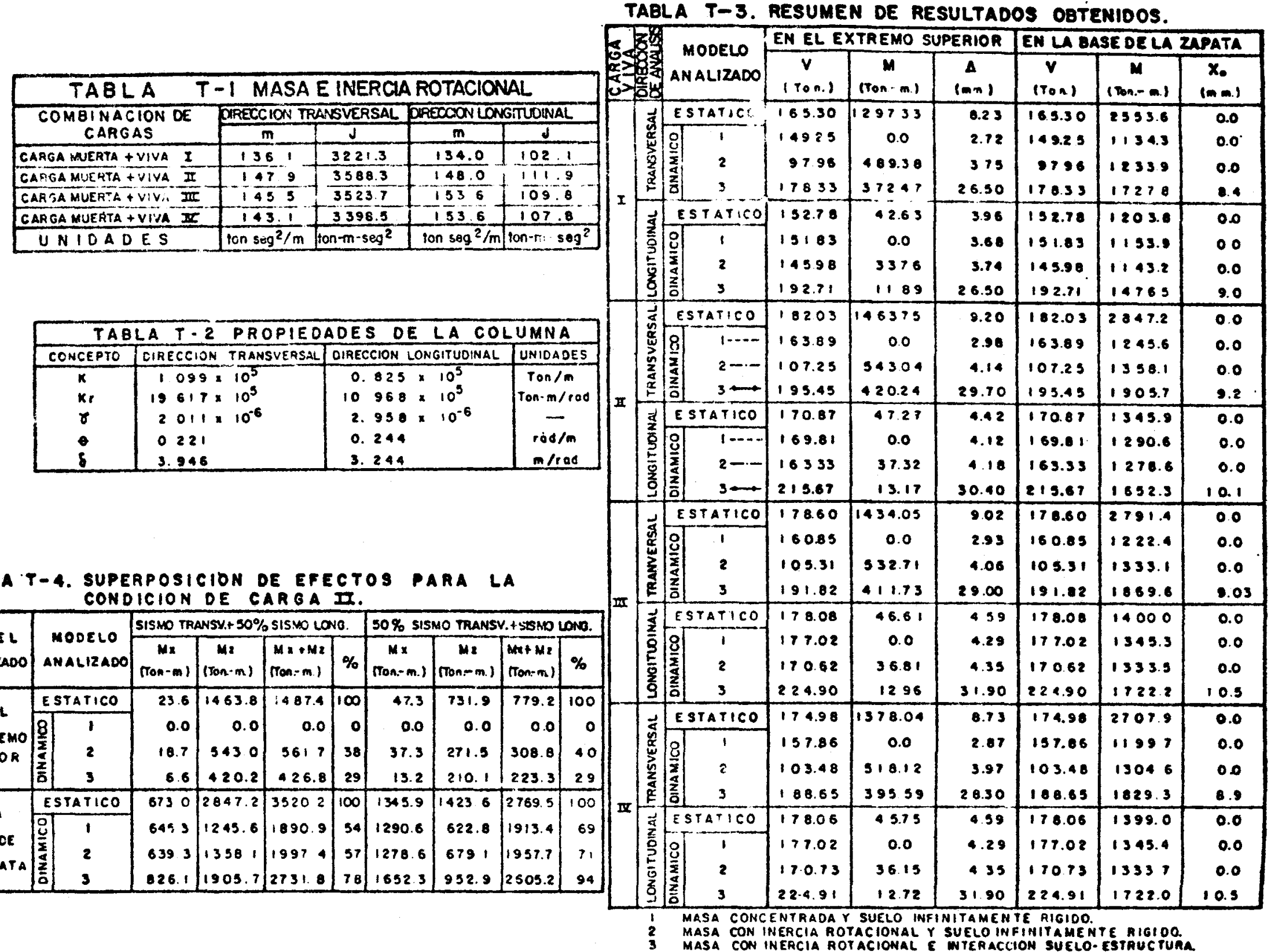

